\title{
The LSST Data Mining Research Agenda
}

\author{
K. Borne*, J. Becla ${ }^{\dagger}$, I. Davidson**, A. Szalay ${ }^{\ddagger}$ and J. A. Tyson ${ }^{\S}$ \\ ${ }^{*}$ Computational and Data Sciences, George Mason University, MS 6A2, Fairfax, VA 22030 \\ ${ }^{\dagger}$ Stanford Linear Accelerator Center, Stanford University, Stanford, CA 94309 \\ ** Department of Computer Science, University of California, Davis, CA 95616 \\ ${ }^{*}$ Department of Physics \& Astronomy, Johns Hopkins University, Baltimore, MD 21228 \\ ${ }^{\S}$ Physics Department, University of California, Davis, CA 95616
}

\begin{abstract}
We describe features of the LSST science database that are amenable to scientific data mining, object classification, outlier identification, anomaly detection, image quality assurance, and survey science validation. The data mining research agenda includes: scalability (at petabytes scales) of existing machine learning and data mining algorithms; development of grid-enabled parallel data mining algorithms; designing a robust system for brokering classifications from the LSST event pipeline (which may produce 10,000 or more event alerts per night); multi-resolution methods for exploration of petascale databases; indexing of multi-attribute multi-dimensional astronomical databases (beyond spatial indexing) for rapid querying of petabyte databases; and more.
\end{abstract}

Keywords: catalogs - surveys - methods: data analysis - astronomical data bases: miscellaneous PACS: $95.80 .+p, 95.75 . P q$

\section{DATA-INTENSIVE ASTRONOMY AND THE LSST SKY SURVEY}

The development of models to describe and understand scientific phenomena has historically proceeded at a pace driven by new data. The more we know, the more we are driven to tweak or to revolutionize our models, thereby advancing our scientific understanding. This data-driven modeling and discovery linkage has entered a new paradigm [1]. The acquisition of scientific data in all disciplines is now accelerating and causing a nearly insurmountable data avalanche [2]. In astronomy in particular, rapid advances in three technology areas (telescopes, detectors, and computation) have continued unabated [3] - all of these advances lead to more and more data [4]. With this accelerated advance in data generation capabilities, we will require novel, increasingly automated, and increasingly more effective scientific knowledge discovery systems [5].

Astronomers have been doing data mining for centuries: "the data are mine, and you can't have them!". Seriously, astronomers are trained as data miners, because we are trained to: (a) characterize the known (i.e., unsupervised learning, clustering); (b) assign the new (i.e., supervised learning, classification); and (c) discover the unknown (i.e., semi-supervised learning, outlier detection) $[6,7,8]$. These skills are more critical than ever since astronomy is now a data-intensive science, and it will become even more data-intensive in the coming decade [4, 9, 10]. New surveys may produce hundreds of terabytes (TB) up to 100 (or more) petabytes (PB) both in the image data archive and in the object catalogs (databases). Discovering the ensuing hidden wealth of new scientific knowledge will require more sophisticated algorithms and networks that discover, integrate, and learn from distributed petascale databases more effectively [11], [12]. 
The problem therefore is this: astronomy researchers will soon (if not already) lose the ability to assimilate or to keep up with any of these things: the data flood, the scientific discoveries buried within, the development of new models of those phenomena, and the resulting new data-driven follow-up observing strategies that are imposed on telescope facilities to collect new data needed to validate and augment new discoveries.

One of the most impressive astronomical sky surveys being planned for the next decade is the Large Synoptic Survey Telescope project (LSST at www.lsst.org) [13]. The three fundamental distinguishing astronomical attributes of the LSST project are:

1. Repeated temporal measurements of all observable objects in the sky, corresponding to thousands of observations per each object over a 10-year period, expected to generate 10,000-100,000 alerts each night to the astronomical research community that something has changed at that location on the sky: either the brightness or position of an object, or the serendipitous appearance of some totally new object;

2. Wide-angle imaging that will repeatedly cover most of the night sky within 3 to 4 nights (= tens of billions of objects); and

3. Deep co-added images of each observable patch of sky (summed over 10 years: 2015-2025), reaching far fainter objects and to greater distance over more area of sky than other sky surveys [14].

Compared to other astronomical sky surveys, the LSST survey will deliver time domain coverage for orders of magnitude more objects. It is envisioned that this project will produce $\sim 30 \mathrm{~TB}$ of data per each night of observation for 10 years. The final image archive will be greater than 60 PB (and possibly much more), and the final LSST astronomical object catalog (object-attribute database) is expected to be $\sim 10-20 \mathrm{~PB}$ (or more). Additional information about the LSST survey and scientific program are described by Ivezic et al. [15] and provided elsewhere in these proceedings [16].

Since it is anticipated that LSST will generate many thousands (probably tens of thousands) of new astronomical event alerts per night of observation, there is a critical need for innovative follow-up procedures. These procedures necessarily must include modeling of the events - to determine their classification, time-criticality, astronomical relevance, rarity, and the scientifically most productive set of follow-up measurements. Rapid time-critical follow-up observations, with a wide range of time scales from seconds to days, are essential for proper identification, classification, characterization, analysis, interpretation, and understanding of nearly every astrophysical phenomenon (e.g., supernovae, novae, accreting black holes, microquasars, gamma-ray bursts, gravitational microlensing events, extrasolar planetary transits across distant stars, new comets, incoming asteroids, trans-Neptunian objects, dwarf planets, optical transients, variable stars of all classes, and anything that goes "bump in the night") [17, 18].

\section{Petascale Mining of Large Astronomical Sky Surveys}

LSST and similar large sky surveys have enormous potential to enable countless astronomical discoveries. Such discoveries will span the full spectrum of statistics: from rare one-in-a-billion (or one-in-a-trillion) type objects, to a complete statistical 
and astrophysical specification of a class of objects (based upon millions of instances of the class). One of the key scientific requirements of these projects therefore is to learn rapidly from what they see. This means: (a) to identify the serendipitous as well as the known; (b) to identify rare events that our models say should be there; (c) to identify new classes of objects that fall outside the bounds of model expectations; (d) to find new attributes of known classes; (e) to provide statistically robust tests of existing models; and (f) to generate the vital inputs for new models. All of this requires integrating and mining all known data: to train classification models and to apply classification models.

LSST alone is likely to throw such data mining and knowledge discovery efforts into the petascale realm. For example: astronomers currently discover a few hundred new supernovae per year. Since the beginning of human history, perhaps $\sim 10,000$ supernovae have been recorded. Because the identification, classification, and analysis of supernovae enable fundamental (Dark Energy) science, it is imperative for astronomers to respond quickly to each new event with rapid follow-up observations in many measurement modes (light curves; spectroscopy; and images of the host galaxy and its environment). Historically, with $<10$ new supernovae being discovered each week, such follow-up has been feasible. But now, LSST promises to produce a list of 1000 new supernovae each night for 10 years [14], which represent a small fraction of the total (10-100 thousand) alerts expected each night! Astronomers are faced with the enormous challenge of efficiently mining, correctly classifying, and intelligently prioritizing a staggering number of new events for follow-up observation each night for a decade.

The major features and contents of the LSST scientific database include: $>100$ database tables; image metadata (675M rows); source catalog (260B rows); object catalog (22B rows, with 200+ attributes); moving object catalog; variable object catalog; alerts catalog; calibration metadata; configuration metadata; processing metadata; and provenance metadata. The science archive will consist of $\sim 2000$ images per night (for 10 years), comprising 60-100 PB of pixel data. This enormous LSST data archive and object database enables a diverse multidisciplinary research program: astronomy \& astrophysics; machine learning (data mining); exploratory data analysis; XLDB (extremely large databases); scientific visualization; computational science \& distributed computing; and inquiry-based science education (using data in the classroom).

Many possible scientific data mining use cases are anticipated with the LSST database, including:

- Provide rapid probabilistic classifications for all 10,000 LSST events each night;

- Find new "fundamental planes" of correlated astrophysical parameters (e.g., the fundamental plane of Elliptical galaxies) [19];

- Find new correlations, associations, relationships of all kinds from 100+ attributes in the LSST science database, integrated with distributed VO-accessible data;

- Compute multi-point multi-dimensional correlation functions over the full panoply of astrophysical parameter spaces;

- Discover zones of avoidance in interesting parameter spaces (e.g., period gaps);

- Discover new properties of known classes;

- Discover new and improved rules for classifying known classes of objects (e.g., photometric redshifts) [20]; 
- Discover new and exotic classes of astronomical objects;

- Identify novel, unexpected temporal behavior in all classes of objects [17];

- Hypothesis testing - verify existing (or generate new) astronomical hypotheses with strong statistical confidence, using millions of training samples;

- Serendipity - discover rare one-in-a-billion objects through novelty detection;

- Image processing - identify non-astronomical features, classify them, and separate them from the astronomical catalog inputs [21, 22]; and

- Quality assurance - identify system glitches, instrument anomalies, and pipeline errors through near-real-time deviation detection.

Some of the data mining research challenge areas posed by the arrival of petascale scientific databases include:

- indexing and associative memory techniques (trees, graphs, networks) for multiattribute (highly-dimensional) astronomical databases (beyond RA-Dec indexing);

- scalability of statistical, computational, machine learning, and data mining algorithms to multi-petabyte scales;

- algorithms for optimization of simultaneous multi-point fitting across massive multi-dimensional data cubes;

- multi-resolution methods and structures for exploration of petascale databases;

- petascale analytics for visual exploratory data analysis of massive databases; and

- rapid query, search, and retrieval algorithms for petabyte databases.

Additional and more in-depth discussion of the petascale data challenges posed by the LSST sky survey are available (at www.lsst.org/Project/docs/data-challenge.pdf and universe.ucdavis.edu/docs/LSST_petascale_challenge.pdf).

\section{A Classification Broker for Astronomy}

We envision an astroinformatics (data-intensive astronomy) research paradigm (for data integration and mining) to address the petascale needs of large astronomical surveys [8, 23]. The impending data loads surpass those of the Sloan Digital Sky Survey by 100010,000 times, while the time-criticality requirement (for event/object classification and characterization) drastically drops from months (or weeks) down to minutes (or tens of seconds). In addition to the follow-up classification problem (described earlier), we will want to find every possible new scientific discovery (pattern, correlation, relationship, outlier, new class, etc.) buried within these new enormous databases. This might lead to a petascale data mining compute engine that runs in parallel alongside the data archive - to test every possible N-point correlation, multi-parameter association, and classification rule. In addition to such a "batch discovery machine", a rapid-response data mining engine (i.e., classification broker) is needed in order to produce and distribute scientifically robust near-real-time classifications of astronomical sources, events, objects, or event host objects (e.g., we need the redshift of the host galaxy in order to interpret and classify a supernova accurately) [23, 24, 25]. These classifications are derived from 
integrating and mining data, information, and knowledge from multiple distributed VOaccessible data repositories, robotic telescopes, and astronomical alert networks worldwide. Incoming event alert data will be subjected to a suite of machine learning (ML) algorithms for event classification, outlier detection, object characterization, and novelty discovery [18, 23, 24, 25, 26, 27]. Probabilistic ML models will produce rank-ordered lists, to guide follow-up observations on the $10-100 \mathrm{~K}$ alertable astronomical events that will be identified each night by the LSST sky survey alone. The classification broker will thereby enable rapid follow-up science for the most important and exciting astronomical discoveries of the coming decade, on a wide range of time scales from seconds to days, corresponding to a plethora of exotic astrophysical phenomena.

\section{ACKNOWLEDGMENTS}

We thank our LSST (www.lsst.org) collaborators for their valuable contributions.

\section{REFERENCES}

1. Mahootian, F., \& Eastman, T. 2008, “Complementary Frameworks of Scientific Inquiry: HypotheticoDeductive, Hypothetico-Inductive, and Observational-Inductive,” World Futures journal, in press.

2. Bell, G., Gray, J., \& Szalay, A. 2005, arxiv.org/abs/cs/0701165.

3. Gray, J., \& Szalay, A. 2004, Microsoft technical report MSR-TR-2004-110.

4. Becla, J., et al. 2006, arxiv.org/abs/cs/0604112.

5. Borne, K. D. 2006, "Data-Driven Discovery through e-Science Technologies," in the proceedings of the 2nd IEEE Conference on Space Mission Challenges for Information Technology.

6. Borne, K. D. 2001a, astro-ph/0008307.

7. Borne, K. D. 2001b, astro-ph/0010583.

8. Borne, K. D. 2009, "Scientific Data Mining in Astronomy," in Next Generation Data Mining, CRC Press, in press.

9. Brunner, R. J., et al. 2001, astro-ph/0106481

10. Szalay, A. S., Gray, J., \& VandenBerg, J. 2002, arxiv.org/abs/cs/0208013.

11. Gray, J., et al. 2002, arxiv.org/abs/cs/0202014.

12. Longo, G., et al. 2001, "Advanced Data Mining Tools for Exploring Large Astronomical Databases," Proceedings of the SPIE, volume 4477, Astronomical Data Analysis, p. 61.

13. Tyson, J. A. 2004, “The Large Synoptic Survey Telescope: Science \& Design,” downloaded from http://www.lsst.org/Meetings/CommAccess/abstracts.shtml.

14. Strauss, M. 2004, "Towards a Design Reference Mission for the LSST," downloaded from http://www.lsst.org/Meetings/CommAccess/abstracts.shtml

15. Ivezic, Z., et al. 2008a, astro-ph/0805.2366.

16. Ivezic, Z., et al. 2008b, "Parametrization and Classification of 20 Billion LSST Objects," in the proceedings of Classification and Discovery in Large Astronomical Surveys, New York: AIP.

17. Paczynski, B. 2000, Publications of the ASP, 112, p. 1281.

18. Becker, A., Astronomische Nachrichten, 329, p. 280.

19. Djorgovski, S., \& Davis, M. 1987, Astrophysical Journal, 313, p. 59.

20. Way, M. J., \& Srivastava, A. N. 2006, Astrophysical Journal, 647, p. 102.

21. Salzberg, S., et al. 1995, Publications of the ASP, 107, p. 279.

22. Waniak, W. 2006, Experimental Astronomy, vol. 21, issue 3, p. 151.

23. Borne, K. D. 2008, Astronomische Nachrichten, 329, p. 255.

24. Bloom, J. S., et al. 2008, Astronomische Nachrichten, 329, p. 284.

25. Mahabal, A., et al. 2008, Astronomische Nachrichten, 329, p. 288.

26. Ball, N. M., et al. 2006, Astrophysical Journal, 650, p. 497.

27. Ball, N. M., et al. 2007, Astrophysical Journal, 663, p. 774. 